\title{
DEVELOPMENT OF A NOVEL SIMULATION CODE TO PREDICT THREE-DIMENSIONAL NEUROGENESIS BY USING MULTILAYERED CELLULAR AUTOMATON
}

\author{
EIJI NAKAMACHI ${ }^{1}$, AKIE NAKAYAMA $^{1}$, TAKEHIKO YAMAMOTO ${ }^{2}$, YUSUKE MORITA $^{1}$ \& \\ HIDETOSHI SAKAMOTO ${ }^{1}$ \\ ${ }^{1}$ Doshisha University, Japan. \\ ${ }^{2}$ Osaka Electro-Communication University, Japan.
}

\begin{abstract}
In this study, a novel simulation code to predict three-dimensional (3D) neurogenesis was developed by using a multilayered cellular automaton (CA) method. Recently, the induced pluripotent stem cell therapy treatments have rapidly grown up as an attractive repair and regeneration technologies for damaged central nervous system (CNS). However, understanding the repair mechanism and developing a numerical analysis code to predict CNS neurogenesis process have ultimate difficulties because more than hundreds of billions of neurons connect each other, and it is almost impossible to analyze the neurogenesis evolution process. Especially, the axonal extension to generate the neural network system is extremely difficult. In this study, based on the phase contrast microscopy (PCM) and the multiphoton microscope (MPM) observations of two-dimensional (2D) and 3D nerve cell network generation of the pheochromocytoma cells (PC12), a novel simulation code to predict the CNS morphogenesis was developed. At first, time-lapse PCM observations have been executed to understand the nerve cell axonal extension and branching. Secondly, 3D representative volume elements (RVEs) of cortex were derived by using Nissl-stained cerebral cortex images. Finally, a 3D CA simulation code for neurogenesis was developed based on multilayered CA algorithms, such as the dendrites outgrowth, an axon selection from dendrites, the extension enhancement induced by the nerve growth factor (NGF), and the branching caused by microtubule collapse under the effect of Netrin-1. Our newly developed CA simulation code was confirmed as a comprehensive code to predict neurogenesis processes through comparison with PCM and MPM observation results.
\end{abstract}

Keywords: cellular automaton, central nervous system, nerve axonal extension, neurogenesis, representative volume element, $3 D$ simulation.

\section{INTRODUCTION}

In this study, a novel simulation code to predict the morphogenesis of the central nervous system (CNS) using a multilayered cellular automaton (CA) method is newly developed.

The neurogenesis process consists of the dendrites outgrowth, the axonal extension, branching and connection with the targeted dendrite of cell body [1-3]. If this neurogenesis process can be predicted by a numerical simulation code, it will not only elucidate the neural network generation, but also contribute to the regeneration technology development of injured CNS. The axonal extension could be predicted by the stochastic analysis technique, such as the phase field theory, Monte-Carlo method and CA method [4-6]. CA is considered superior, because it utilizes the simple local neighborhood rule and transition rule. With the using of the CA method, it is possible to express phenomena without finding the governing equations, so the synthesis and migration of proteins involved in neuronal axon formation and extension within cells are introduced into CA algorithm easily. Furthermore, the neurogenesis process can be simulated by using stacking rules based on the interaction between cells. Using the multilayered CA method, the influences of intra- and extracellular matrices (ICM and ECM) on the axonal extension and neurogenesis process, such as the densities of shooting 1, the nerve growth factor (NGF) and Netrin-1 and the stress field, are predicted 
rationally [7]. Two-dimensional (2D) and three-dimensional (3D) CA models are formulated based on the experimental observation results of the phase contrast microscope (PCM) and the multiphoton microscope (MPM).

However, these numerical codes were not verified through comparison with in-vivo observations. Furthermore, it is difficult to conduct a large-scale simulation taking into consideration the large number of neurons contained in the whole brain region. Therefore, in this study, a representative volume element (RVE) of cerebral cortex is derived, and the neurogenesis process simulations using a multilayered CA method is carried out.

Main issues in this study are as follows:

1. Experimental observations of nerve axonal extension process.

2. Formulation of multilayered CA model.

3. Neurogenesis process simulations in $2 \mathrm{D}$ and $3 \mathrm{D}$.

\section{EXPERIMENTS}

At first, time-lapse microscopic observations of the pheochromocytoma cells (PC12) through the PCM (Olympus Co. IX73) were carried out in order to analyze nerve axonal extension processes in $2 \mathrm{D}$ region and the axon morphologies in the $3 \mathrm{D}$ region. $\mathrm{PC} 12$ were supplied by the System Life Science Laboratory at the Department of Medical Life System at Doshisha University. The cells were cultured in Dulbecco's modified Eagle's medium supplemented with $10 \%$ horse serum, $5 \%$ fetal bovine serum and $0.05 \%$ antibiotic in each collagen-coated $35 \mathrm{~mm}$ dish in a $5 \% \mathrm{CO}_{2}$ incubator at $37^{\circ} \mathrm{C}$ with an addition of NGF $(50 \mathrm{ng} / \mathrm{ml})$, at 0 days. The PC12 suspension density is 625 cells $/ \mathrm{cm}^{2}$.

Figure 1 shows the axonal extension process of PC12 captured by the time-lapse PCM observation. Images of the neurogenesis process were captured every $1 \mathrm{~min}$ for $24 \mathrm{~h}$ after the addition of NGF. At the early stage of cultivation, a PC12 has a number of short dendrites. Thereafter, a certain dendrite becomes an axon, and the nerve cell is polarized. The experimental observation indicated that the average number of dendrites was 3.6, and the dendrites appeared randomly on the outer periphery of a cell body. Furthermore, the average extension rate of the nerve axon was $20.4 \mu \mathrm{m} / \mathrm{h}$, which is similar to the result for a single cell $(21.9 \mu \mathrm{m} / \mathrm{h})$ in the experiments by Nakamachi et al. [8].

Toriyama and Shimada identified a protein shootin1, which increases at the nerve cell polarity stage, concentrates at the tip of dendrites, and works to enhance the nerve axonal extension in the upstream region of signal molecules that enhance the dendrite extension. The shootin1 localized in a 'wave' structure after it was synthesized in the nerve cell body and was transported from the nerve cell body to the tip of the nerve axon with the wave [9-11]. Both the transportation amount of shootin 1 and the period of the wave occurrence are completely random. The diffusion speed in a short dendrite is considered to be faster than that in a long dendrite, because the concentration gradient of short dendrites is larger than that of long dendrites. Consequently, longer dendrites maintain a higher concentration of shootin 1 and hence promote the selected nerve axon's extension more remarkably [12, 13]. In the axonal extension process, there occurred the branching under the Netrin-1 stimulation $[14,15]$. It was also reported that the enhancement of axonal extension was promoted by the NGF density gradient depending on the distance from the target nerve cell [16-18].

Figure 2 shows 3D and top view of PC12 morphology by using MPM (Leica, TCS SP8 MP). PC12 cell bodies, dendrites and axons were observed. The axonal extension, connection and network generation were featured in such a way that the extension is affected by the distance between the cell body and its targeted cell body. 

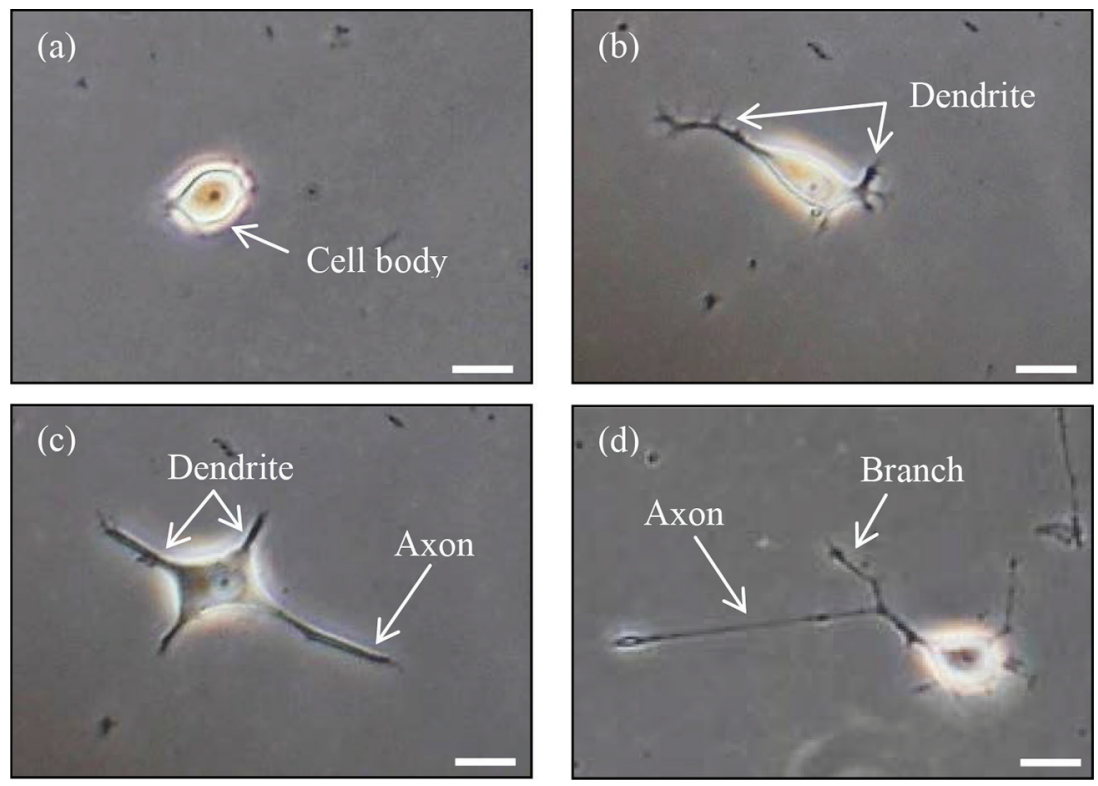

Figure 1: Photos of cultivated PC12 after the addition of NGF (Bar: $10 \mathrm{~mm}$ ); (a) 3h, (b) 6h, (c) $12 \mathrm{~h}$ and (d) $24 \mathrm{~h}$.

(a)

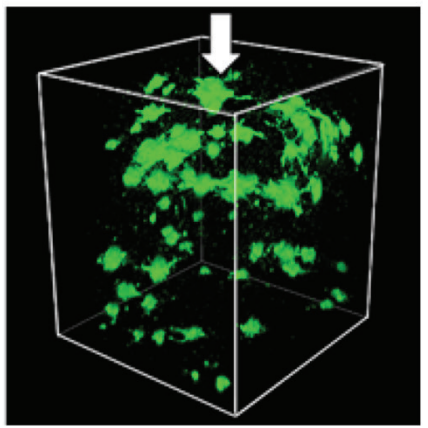

(b)

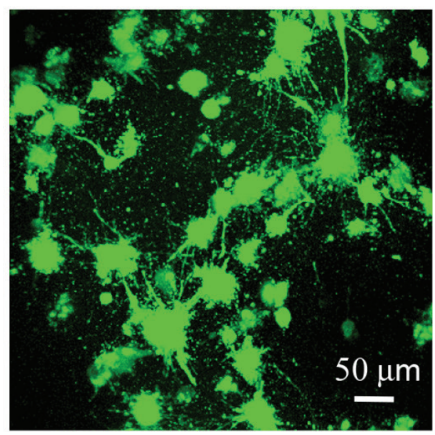

Figure 2: MPM images of PC12 cell bodies, dendrites, and axons. (a) 3D image of 500 $\mu \mathrm{m}$ cube region and (b) top view of cell bodies and axons.

\section{CA ALGORITHM}

A multilayered CA method to predict pc12 morphogenesis by introducing the dendrite and axonal extension is developed based on the experimentally observed results. Without solving the governing equation of the simulative objects, CA can predict the complex dynamic morphogenesis of a nerve cell by adopting the simple local neighborhood rules and transition rules. In a CA method, the simulation region is divided into the cell (unit cell of CA model), such as the quadrate, $2 \mathrm{~d}$, and the cube, $3 \mathrm{~d}$. Each cell has an arbitrarily selected condition and changes its condition according to the neighborhood cells' condition. The change of state parameter is decided based on local rules. The change of cell condition occurs for all cells simultaneously. 


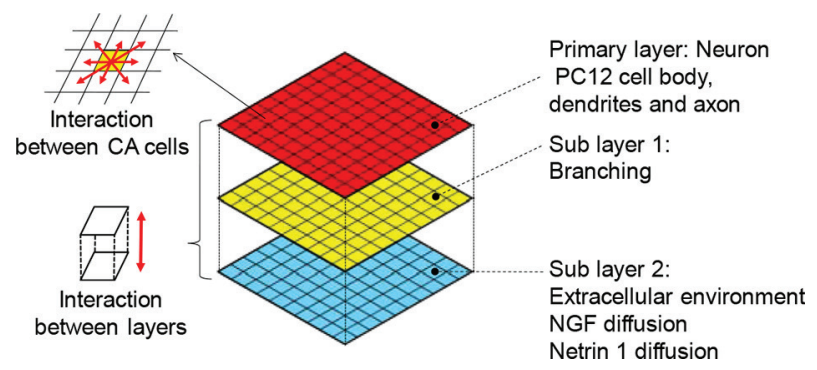

Figure 3: Schematic view of a multilayered CA model, cells and interactions.

The condition at a given cell and time is determined depending on the self-determined condition and neighborhood cells' condition. Moore's CA model is adopted in our CA calculations [5-6].

A multilayered CA method to consider the interactions between nerve axon and various extracellular environmental stimulations and the cell constituents is formulated and implemented in CA code. Figure 3 shows a schematic view of a multilayered CA model, which consists of a primary layer of dendrites and axonal extensions, a 'sub-layer 1' of axon branching and a 'sub-layer 2' of ngf and netrin-1 diffusions. In these layers, there occurs the interaction between cells and layers as shown in Fig. 3.

3.1 Nerve cell's axonal extension and NGF diffusion models in the primary layer and the Sub-layer 2

The dendrites outgrowth and the selection of axon are formulated in the primary layer as shown in Fig. 3. The primary layer has feature that the nerve dendrite extension is depends on the its length: the longer the length of dendrites once occurs, the faster the extension speed and finally only one dendrite will be selected as the axon as shown in Fig. 4.

In the following, we formulated a CA simulation model for nerve axonal extension considering the time-lapse observation results. The extension of dendrites is enhanced depending on
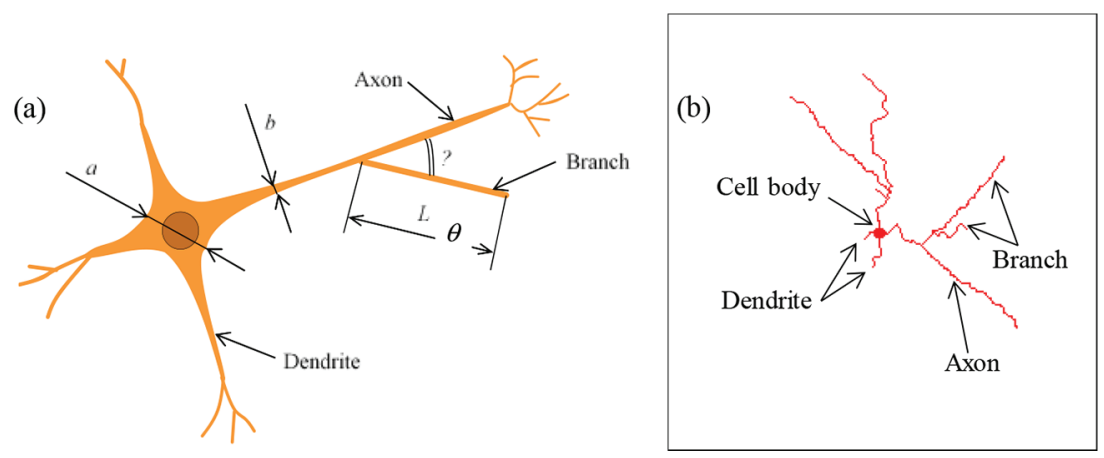

Figure 4: Schematic view of axon morphology model: (a) characteristic values of PC12 nerve cell determined based on experimental observation; $a$ the diameter of the cell body, $b$ the width of the axon, $L$ the length of branch, $\theta$ deviation angle of the branch from the axon. (b) An example of axon extension with branch. 
the concentration of shootin 1 around an axon tip. Shootin 1 moves randomly inside a nerve cell, and a dendrite that stores more shootin1 extends more rapidly. Consequently, an axon that extends ahead of other dendrites tends to grow more remarkably because longer axon has higher concentrations of shootin1 [9-11].

In order to represent these phenomena in the CA simulation, a dendrite that grows in the next time step was decided according to a probability function depending on the dendrite length. The outgrowth probability function $P_{\mathrm{n}}$ of $n_{\mathrm{th}}$ dendrite is defined by

$$
P_{n}=\frac{a^{l_{n}}}{\sum_{k=1}^{m} l_{k}}
$$

where $m$ is the total number of dendrites, $l_{k}$ denotes the length of dendrite $k$ scaled by the length of the computational cell side, and $a$ is a random parameter, which was set to 1.08 in the present simulation considering the experimental results.

After the decision to extend a dendrite in the next step, the direction of its extension was stochastically chosen at each time step from three candidates, as shown in Fig. 5(a) and (b), in the cases of 2D and 3D regions, according to a normal random number with an average of 0 and a variance of 1 by using the normal distribution probability function. These selection processes of the dendrite and the decision of the extension direction were repeated in predetermined time steps.

NGF released from arbitrary cells was assumed to follow the diffusion equation as given by

(a)

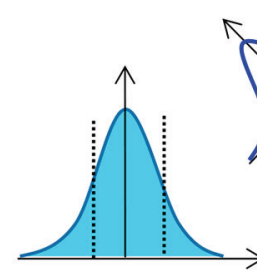

Standard Gaussian distribution

(b)

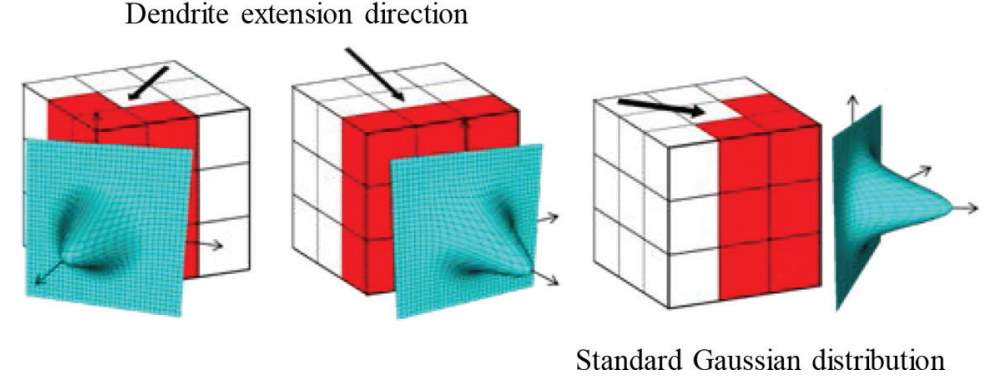

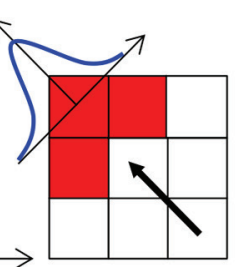

Dendrite extension direction

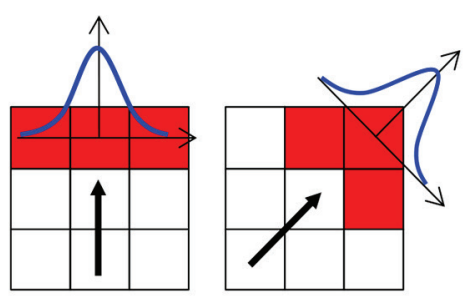
Dendrite extension direction

Figure 5: Dendrite extension direction determination algorithm using the normal distribution probability function in cases of $2 \mathrm{D}$ and $3 \mathrm{D}$ regions: (a) $2 \mathrm{D}$ and (b) $3 \mathrm{D}$. 


$$
\frac{\partial c}{\partial t}=D\left(\frac{\partial^{2} c}{\partial x^{2}}+\frac{\partial^{2} c}{\partial y^{2}}+\frac{\partial^{2} c}{\partial z^{2}}\right)
$$

Here, $c$ is the concentration, $t$ the time, $D$ the diffusion coefficient and $(x, y, z)$ the coordinate of an arbitrary point. In our CA model, we adopted the value of $D$ as $78 \mu \mathrm{m}^{2} / \mathrm{s}$. The total amount of NGF contained in the cells changes over time, and the NGF concentration $c(x$, $y, z)$ at time $t$ at an arbitrary point $(x, y, z)$, where $N$ cells exist in the simulation region, is given by

$$
c(x, y, z, t)=\sum_{i=1}^{N} \frac{C_{i}(t)}{(\sqrt{4 \pi D t})^{3}} e^{-\frac{\left(x-x^{\prime}\right)^{2}+\left(y-y^{\prime}\right)^{2}+\left(z-z^{\prime}\right)^{2}}{4 D t}}
$$

where the central coordinate of the $i$-th cell body is $\left(x^{\prime}, y^{\prime}, z^{\prime}\right)$ and the NGF concentration at the center is $C_{i}(t)$.

In accordance with the physiological characteristics obtained by eqns. (1)-(3) for the neurons, we modeled the process by which cells extend the nerve axons and search for target dendrites. First, each cell body contains four dendrites in each PC12 cell body in the case of 2D model and six dendrites in 3D model. The nerve axons detect the NGF concentration at the terminal and extend in the direction of higher concentration. The NGF concentration that the nerve axon can detect is $133 \mathrm{ng} / \mathrm{ml}$ or more, and when it exceeds $995 \mathrm{ng} / \mathrm{ml}$, receptor down-regulation occurs, so NGF does not affect the extension of nerve axon no more. When the nerve axon and target dendrite are combined, the concentration of NGF in the somatic cell body on the axon side increased, and, on the other hand, decreased in the dendritic side. Binding was judged as the case where the neuronal axons and the cells at the tip of the dendrites were adjacent.

\subsection{Netrin-1 diffusion model and branch model in sub-layer 2 and 1}

In the 'Sub-layer 2' as shown in Fig. 3, Netrin-1 released from arbitrary cells was assumed to follow the same diffusion equation as discussed in the case of NGF diffusion process simulation. We adopted the value of diffusion coefficient $D$ as $40 \mathrm{~mm}^{2} / \mathrm{s}$ [19]. Furthermore, introducing the interaction with 'Sub-layer 2,' the branching process is formulated in CA model in 'Sub-layer 1.'

When the nerve cell is excited, the impulse signal moves on to the nerve axon. The signal stimulates synaptic vesicles in the synaptic terminal button via the presynaptic terminal, and the neurotransmitter in the vesicle is released into the synaptic cleft, triggering the excitation and suppression of the sub-synaptic membrane. At this time, Netrin-1 is released from the underlying synaptic membrane and its periphery, which is absorbed at the presynaptic terminal and passes through the nerve axon and is retrograde transported into the excitable cell. The speed of retrograde transport is as fast as $200-400 \mathrm{~mm}$ /day for the extension rate of the nerve axon at $20.7 \mu \mathrm{m} / \mathrm{h}[8]$.

In order to represent Netrin-1 enhances axonal extension, a nerve axonal tip cell extends two separated cells under the condition defined by Netrin 1 cells around the targeted cell in CA model. Furthermore, when Netrin-1 concentration around the tip of dendrites is above the threshold level that we set, microtubule collapse to present Netrin-1 promotes axonal branch formation. The concentration of Netrin-1 is calculated by 


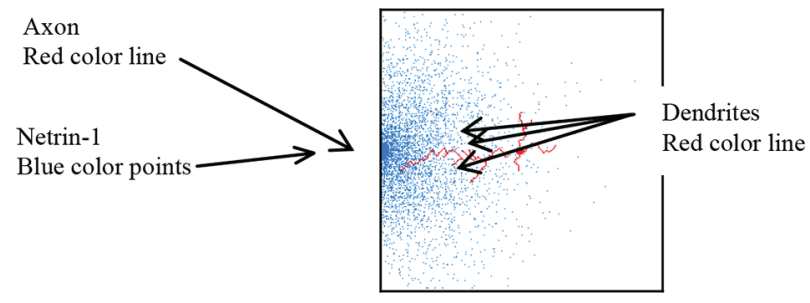

Figure 6: CA results of distribution of Netrin-1 density and axon and dendrites.

$$
C_{n}=\frac{\sum_{l=-5}^{5} \sum_{m=-5}^{5} a_{t}^{i+l, j+m}}{121} \times 100
$$

where $C_{n}$ is a Netrin- 1 concentration around tip of dendrite and $a_{\mathrm{t}}$ is the state variable of each cell. Other rules remain unchanged from the nerve axon extensional model. Fig. 6 shows the diffusion process simulation result of Netrin-1 and axon and dendrites. It shows that the axon is extended with one branch against the high density area of Netrin-1.

Finally, we constructed a multilayered CA model that consists of a nerve axonal extension model (main layer), microtubule elongation and branching model (Sub-layer 1) and NGF diffusion model (Sub-layer 2) as shown in Fig. 3.

\section{RESULTS AND DISCUSSION}

Figure 7(a-1), (b-1), (c-1) and (d-1) shows the results of simple nerve axonal extension simulation in indicated CA calculation steps. In this simulation, extracellular environmental factor, such as Netrin-1, and microtubule as discussed in Section 3.2 were not considered. We successfully represent the phenomenon that four dendrites are generated from cell body and only one dendrite extends as a nerve axon. We conducted the simulation multiple times and confirmed that selection of axonal extension direction was non-deterministic. Fig. 8 shows comparison with CA calculation results and PCM observation results in both four cases of single PC12 cell bodies and axonal extensions. It shows good agreement of axonal extension morphologies.

As discussed in Section 3.2, Netrin-1 enhanced the dendrites extension and promoted the selection of axon. Simultaneously, branches were formed in dendrites and nerve axons due to microtubule collapse in the Sub-layer 2. Furthermore, Netrin 1 promoted the catastrophe of the microtubule, so the nerve axon on the Netrin-1-rich side has more branches. Fig. 7 (a-2), (b-2), (c-2) and (d-2) shows the results of multilayered CA simulation of nerve axonal extension considering effects of microtubule collapse caused by Netrin- 1 .

Next, 3D RVEs of cortex were derived by using Nissl-stained cerebral cortex images of the mouse living brain obtained from Allen Brain Atlas shown in Fig. 9. Binarization of Nissl stained image using image processing software ImageJ, center coordinates of nerve cells, and cell density in each layer were obtained. Layer II/III, Layer IV/V and Layer VI were selected to characterize the cerebral cortex at the micro scale as shown in Fig. 9. For neurogenesis processes analyses using our multilayered CA models, three RVEs were determined by using the least residual sum of square search to find minimum size of RVE as shown in Fig. 10(a)(c). The objective function was the error of nerve cell density between the population of whole region and the RVE candidate region in three layers. As a result, the sizes of RVEs were all equal to $30 \mu \mathrm{m}$. The number of nerve cells contained in each RVE was calculated as 13,11 and 16, respectively. 

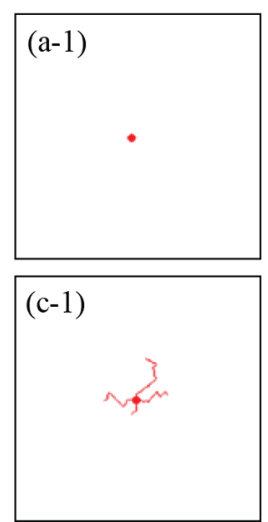
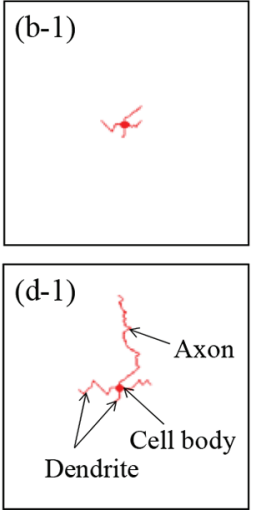

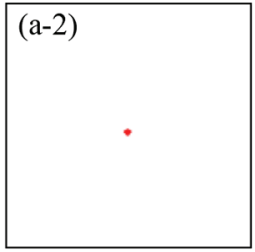

$(\mathrm{c}-2)$

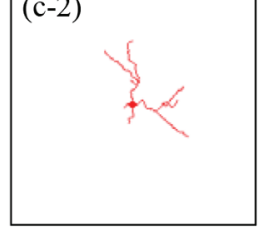

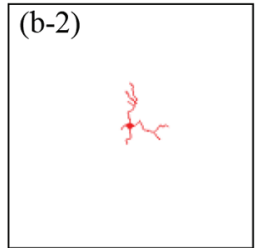

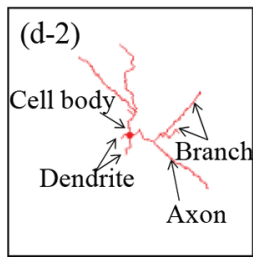

Figure 7: Simulation results of the axonal extension in the arbitrary time steps of without the branch; (a-1) 0 step, (b-1) 40'104 step, (c-1) 80'10 $0^{4}$ step and (d-1) $100^{\prime} 10^{4}$ step, and with the branch; (a-2) 0 step, (b-2) 40¹0 $10^{4}$ step, (c-2) $80^{\prime} 10^{4}$ step and (d-2) $100^{\prime} 10^{4}$ step.

(a)
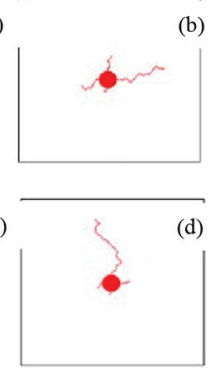

(b)

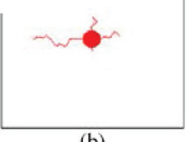

(b)

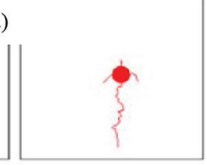

(d) (e)

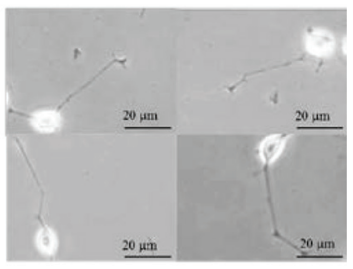

Figure 8: Nerve axonal extensions without branch: CA calculation results (a) 1st (b) 2nd (c) 3rd and (d) 4th results, (e) Experimental results.

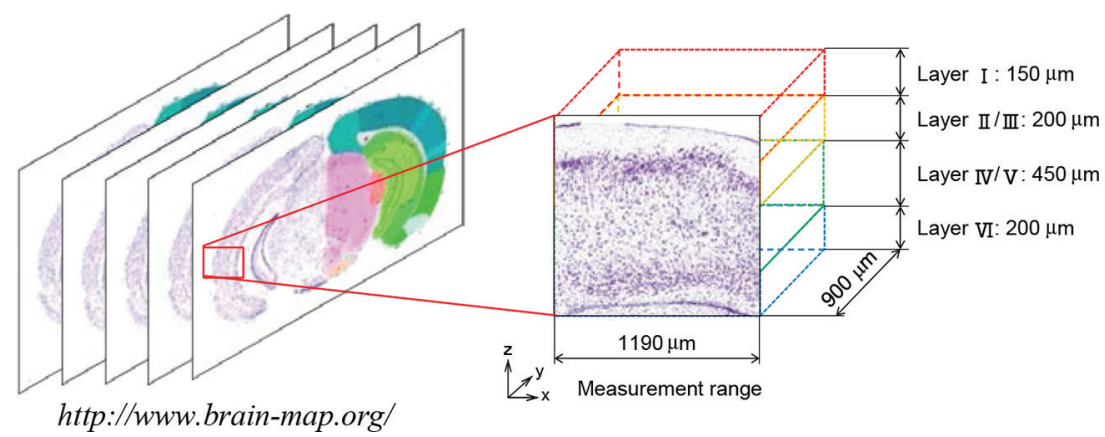

Figure 9: Nissl-stained cerebral cortex images of the mouse living brain obtained from Allen Brain Atlas (http://www.brain-map.org/) (a) scanned images and (b) layers structure. 
(a)

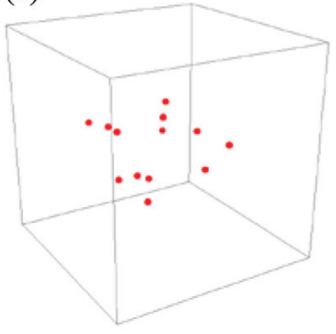

(b)

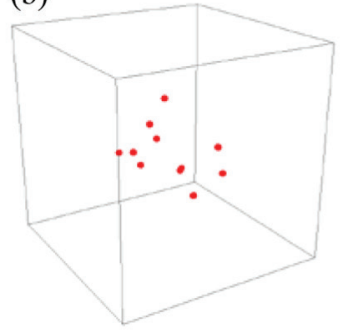

(c)

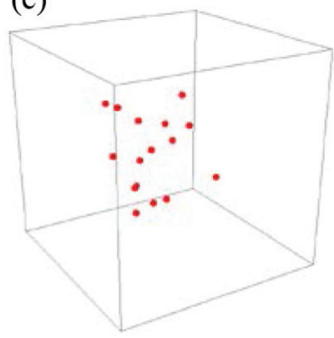

Figure 10: RVEs of nerve cells: (a) layer II/III (b) layer IV/V and (c) layer VI.

(a1)

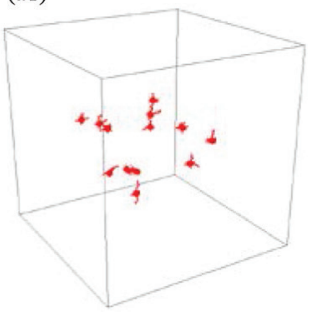

(a2)

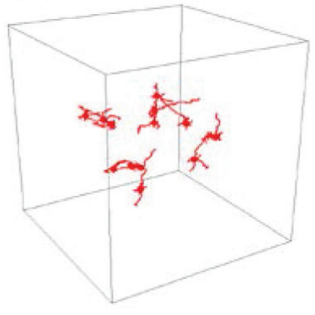

(b1)

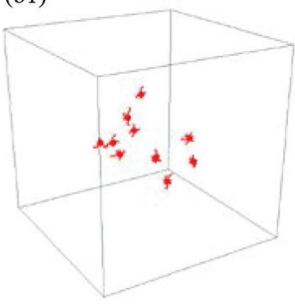

(b2)

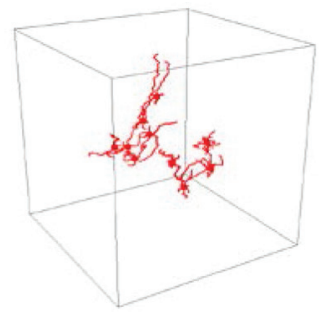

(c1)

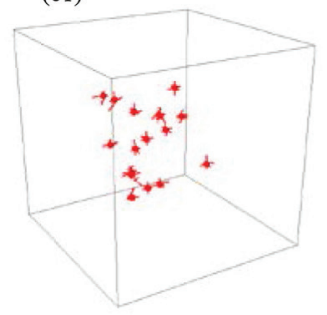

(c2)

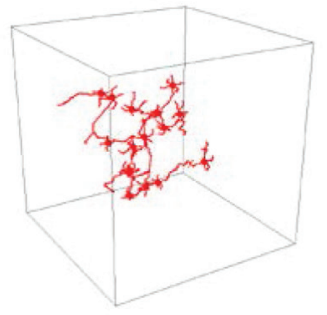

Figure 11: Simulation results of neurogenesis of (a) layer II/III, (b) layer IV/V and (c) layer VI: (a1) 50, (a2) 150, (b1) 50, (b2) 186, (c1)50, (c2) 184 (step number).

Figure 11 shows early and final stages of neurogenesis processes in three layered RVEs. Each layered morphologies show the different distributions of cell bodies and orientations of nerve axons. Especially, the network orientation is strongly related to the signal transmission, such as more inward orientation in the inside layers IV/V and VI, and more in-surface plane orientation in the outside layer II/III.

CA simulation results characterized the network orientation in three layers of mouse cerebral cortex, which relates to the electric signal transmission.

\section{CONCLUSION}

A multilayered CA calculation code was developed to predict the neurogenesis processes in 2D and 3D regions based on experimental observation results by using PCM and MPM. The present model can include intra- and extra-cellular environmental factors such as NGF, 
microtubule and Netrin-1 by adopting the local rules between layers. Accordingly, we successfully restaged nerve axonal extension and branch process considering diffusions of NGF and Netrin-1. Moreover, it is easy to consider additional factors affecting the nerve axonal extension in our newly proposed CA code.

\section{REFERENCES}

[1] Campenot, R.B., Local control of neurite development by nerve growth factor. Proceedings of the National Academy of Sciences, 74(10), pp. 4516-4519, 1997.

[2] Song, H.J. \& Poo, M.M., The cell biology of neuronal navigation. Nature Cell Biology, 3(3), pp. 81-88, 2001.

[3] Marti, M., Sakaguchi, D.S. \& Mallapragada, S., Neural tissue engineering startegies. Regenerative Medicine and Cell Therapy, ed. J.F. Stolz. Ios Press: Washington, DC. 2012, https://doi.org/10.3233/978-1-61499-076-5-275

[4] Takaki, T., Nakagawa, K., Morita, Y. \& Nakamachi, E., Phase-field modeling for axonal extension of nerve cells. Mechanical Engineering Journal, 2(3), 2015, https://doi. org/10.1299/mej.15-00063

[5] Schiff, J.L., Cellular Automata: A discrete view of the world. Wiley-Interscienc, 1, pp. 92-94, 2007.

[6] Ospina, M.E.E. \& Perdomo, J.G., A growth model of human papillomavirus type 16 designed from cellular automata and agent-based models. Artificial Intelligence In Medicine, 57(1), pp. 31-47, 2003, https://doi.org/10.1016/j.artmed.2012.11.001

[7] Bandini, S. \& Mauri, G., Multilayered cellular automata. Theoretical Computer Science, 217(1), pp. 99-113, 1999, https://doi.org/10.1016/s0304-3975(98)00152-2

[8] Nakamachi, E., Yanagimoto, J., Murakami, S. \& Morita, Y., Development of microarray device for functional evaluation of PC12D cell axonal extension ability. Journal of Micro/Nanolithography, MEMS, and MOEMS, 13(2), 2014-6, https://doi.org/10.1117/1. jmm.13.2.023013

[9] Toriyama, M., Shimada, T., Kim, K.B., Mitsuba, M., Nomura, E., Katsuta, K., ... \& Inagaki, N., Shootin1: a protein involved in the organization of an asymmetric signal for neuronal polarization. The Journal of Cell Biology, 175(1), pp. 147-57, 2006, https:// doi.org/10.1083/jcb.200604160

[10] Shimada, T., Toriyama, M., Uemura, K., Kamiguchi, H., Sugiura, T., Watanabe, N. \& Inagaki, N., Shootin1 interacts with actin retrograde flow and L1-CAM to promote axon outgrowth. The Journal of Cell Biology, 181(5), pp. 817-829, 2008, https://doi. org/10.1083/jcb.200712138

[11] Toriyama, M., Sakumura, Y., Shimada, T., Ishii, S. \& Inagaki, N., A diffusion-based neurite length -sensing mechanism involved in neuronal symmetry breaking. Molecular Systems Biology, 6(1), pp. 1-16, 2010, https://doi.org/10.1038/msb.2010.51

[12] Gordon, R. \& Gary, B., Role of moving growth cone-like "Wave" structures in the outgrowth of cultured hippocampal axons and dendrites. Journal of Neurobiology, 39(1), pp. 97-106,1999, https://doi.org/10.1002/(sici)1097-4695(199904)39:1<97::aidneu $8>3.0 . c 0 ; 2-z$

[13] Goodhill, G.J., Diffusion in axon guidance. European Journal of Neuroscience, 9(7), pp. 1414-1421, 1997, https://doi.org/10.1111/j.1460-9568.1997.tb01496.x

[14] Yu, W., Qiang, L., Solowska, J.M., Karabay, A., Korulu, S. \& Baas, P.W., The microtubule-severing proteins spastin and katanin participate differently in the formation of axonal branches. Molecular Biology of the Cell, 19(4), pp. 1485-1498, 2008, https:// doi.org/10.1091/mbc.e07-09-0878 
[15] Tang, F. \& Kalil, K., Netrin-1 induces axon branching in developing cortical neurons by frequency-dependent calcium signaling pathways. Journal of Neuroscience, 25(28), pp. 6702-6715, 2005, https://doi.org/10.1523/jneurosci.0871-05.2005

[16] Ming, G.., Song, H., Berninger, B., Holt, C.E., Tessier-Lavigne, M. \& Poo, M., cAMPdepend growth cone guidance by netrin-1. Journal of Neuron, 19(6), pp. 1225-1235, 1997, https://doi.org/10.1016/s0896-6273(00)80414-6

[17] Robert, B.C., Local control of neurite development by nerve growth factor. Journal of Cell Biology, 74(10), pp. 4516-4519, 1997, https://doi.org/10.1073/pnas.74.10.4516.

[18] Hong-jun, S. \& Mu-ming, P., The cell biology of neuronal navigation. Journal of Nature Cell Biology, 3(3), pp. 81-88, 2001, https://doi.org/10.1038/35060164

[19] Goodhill, G.J., Diffusion in axon guidance. European Journal of Neuroscience, 9(7), pp. 1414-1421, 1997, https://doi.org/10.1038/35060164 